\title{
Forming and Dissolving Partnerships in Cooperative Game Situations
}

Platz, Trine Tornøe; Østerdal, Lars Peter Raahave

Publication date:

2010

Document version

Publisher's PDF, also known as Version of record

Citation for published version (APA):

Platz, T. T., \& Østerdal, L. P. R. (2010). Forming and Dissolving Partnerships in Cooperative Game Situations. Department of Economics, University of Copenhagen. 


\section{Discussion Papers Department of Economics University of Copenhagen}

No. $10-24$

Forming and Dissolving Partnerships

in Cooperative Game Situations

Trine Tornøe Platz, Lars Peter Østerdal

Øster Farimagsgade 5, Building 26, DK-1353 Copenhagen K., Denmark

Tel.: +45353230 01 - Fax: +4535323000

http://www.econ.ku.dk 


\title{
Forming and dissolving partnerships in cooperative game situations*
}

\author{
Trine Tornøe Platz, Lars Peter Østerdal ${ }^{\dagger}$ \\ Department of Economics, University of Copenhagen
}

June 2009, revised September 2010

\begin{abstract}
A group of players in a cooperative game are partners (e.g., as in the form of a union or a joint ownership) if the prospects for cooperation are restricted such that cooperation with players outside the partnership requires the accept of all the partners. The formation of such partnerships through binding aggrements may change the game implying that players could have incentives to manipulate a game by forming or dissolving partnerships. The present paper seeks to explore the existence of allocation rules that are immune to this type of manipulation. An allocation rule that distributes the worth of the grand coalition among players, is called partnership formation-proof if it ensures that it is never jointly profitable for any group of players to form a partnership and partnership dissolution-proof if no group can ever profit from dissolving a partnership. The paper provides results on the existence of such allocation rules for general classes of games as well as more specific results concerning well known allocation rules.
\end{abstract}

\footnotetext{
*Special thanks are due to Peter Holch Knudsen and Mich Tvede for valuable input at the early stages of this work. We also thank Philippe Solal for comments. Of course, all deficiencies remain the responsibility of the authors.

${ }^{\dagger}$ Correspondence: Lars Peter Østerdal, Department of Economics, University of Copenhagen, Øster Farimagsgade 5, Building 26, DK-1353 Copenhagen K, Denmark. Email: lars.p.osterdal@econ.ku.dk. Tel: +45 35323561, Fax: +4535323000.
} 
JEL classification: C71, D63, D71.

Keywords: Cooperative games, partnerships, partnership formation-proof, partnership dissolution-proof.

\section{Introduction}

A problem common to societies, clubs, joint venture organizations, and other formal social structures is how to allocate the benefit, or cost, of a joint activity among the contributing members. In the language of cooperative game theory we can describe such a problem in the following stylized way. There is a set of players and each coalition of players has a worth. This worth can be thought of as the income or surplus that the coalition can obtain without cooperating with the other players, or it can be thought of as a claim on total income. The problem is then to find an allocation rule that specifies how total income (i.e., the worth of the grand coalition) is distributed among the players for any configuration of the coalitional worths.

The present paper is concerned with the players' incentives to create binding agreements - called partnerships - in such cooperative game situations. If a group of players create a partnership, they commit to not cooperate with players outside the partnership without the accept of the rest of the group. We may think of such partnerships as if every player in the group is given veto power over activities involving any member of the partnership. The remaining players outside the partnership are also affected; these outside players are deprived of the possibility of collaborating with any strict subset of the players in the partnership.

Examples of partnerships include members of a parliament joined in political parties or particular parties in a coalition government, a couple getting married, countries forming a union (e.g., a trade union or a political union), workers or groups of workers forming a labor union, partners within a firm, or firms establishing joint ownerships over a common pool of assets.

The creation of a partnership may change the game and hence the outcome of any 
allocation process. Thus the creation or dissolution of a partnership can be seen as a way of changing the cooperative game situation, whether it is due to players seeking to manipulate the game or due to a modelling choice by the analyst. In any case, for someone deciding on an allocation rule to be implemented in a cooperative game situation, knowledge on whether a specific allocation rule gives players incentives to manipulate the game by forming or dissolving partnerships could be highly relevant. Particularly it may be of special interest to consider allocation rules that are "immune" to such manipulation, or at least to be aware of if such rules exist at all.

We shall call an allocation rule partnership formation-proof if it is never strictly profitable for any coalition of players to form a partnership when applying the allocation rule and partnership dissolution-proof if it is never strictly profitable to dissolve a partnership. Thus, implementing a partnership formation-proof allocation rule implies that no manipulation in the form of players forming partnerships will occur while a partnership dissolution-proof allocation rule will be immune to manipulation in the form of players dissolving partnerships. The present paper explores whether allocation rules exist that are in this way immune to manipulation while still satisfying some desirable properties for allocation rules.

Manipulation of cooperative games has been studied by numerous papers starting with, e.g., Postlewaite and Rosenthal (1974), Charnes and Littlechild (1975), Hart and Kurz (1983), Kalai and Samet (1987), Legros (1987), Lehrer (1988), and Hart and Moore (1990). The present paper is closely related to this literature, however, there are also some important differences. In Postlewaite and Rosenthal (1974), Legros (1987), Lehrer (1988), and more recently Haviv (1995), Derks and Tijs (2000), and Knudsen and Østerdal (2008) groups of players can amalgamate into a single player. ${ }^{1}$ The present paper follows Haller (1994), Carreras (1996) and Segal (2003) and considers environments where the set of players is fixed but the worth of coalitions can be manipulated.

Haller (1994) focuses on bilateral agreements (i.e. agreements between two players),

\footnotetext{
${ }^{1}$ Postlewaite and Rosenthal (1974) and Legros (1987) follow Aumann (1973) and refer to a group of players amalgamating into a single player as a syndicate. Charnes and Littlechild (1975) call it a union.
} 
and considers so-called proxy- and association-agreements. In a proxy-agreement one of the players becomes a null player, while the other player's marginal contribution to a coalition is set equal to the two players' joint marginal contribution. ${ }^{2}$ In an association-agreement, if one of the players enter a coalition, it contributes as if both players entered. Carreras (1996) considers partnerships as defined in the present paper and uses the Shapley value to discuss the effect of partnership formation in (especially) simple games. ${ }^{3}$ Segal (2003) contains a general taxonomy of types of integration.

In contrast to e.g. the proxy- and association-agreements discussed by Haller (1994) the creation of partnerships does not yield any technical efficiency gains as a partnership does not increase the worth of any coalition as long as the game is monotonic; it only reduces the worth of coalitions containing some but not all members of the partnership. On the other hand, there is a dual effect of creating a partnership: Players outside the partnership cannot obtain the full worth from cooperation with strict subsets of players in the partnership. The purpose of forming a partnership should therefore be to reduce the power of outside players without reducing the power of players in the partnership equally. However, it is generally not clear which of the aforementioned effects dominates.

Section 2 introduces the model and basic definitions. In section 3 some results on the existence of partnership formation- and dissolution-proof allocation rules are given for several general classes of cooperative games. We find that the equal-split rule is the only symmetric allocation rule that is both partnership formation-proof and partnership dissolution-proof. Further, while no symmetric partnership formation-proof allocation rule exists that satisfies the null player condition we find that on the class of monotonic games $^{4}$ there do exist symmetric partnership dissolution-proof allocation rules satisfying the null player condition. In section 4 we restrict attention to convex games and consider some well-known allocation rules with favorable properties on this class of games. We

\footnotetext{
${ }^{2}$ Haller (1994, Section 6.4) discusses the similarities and differences between proxy-agreements and amalgamations.

${ }^{3}$ See also Carreras et al. (2005), Carreras et al. (2009).

${ }^{4}$ Monotonic games hold the property that no player contributes negatively to a coalition.
} 
find that while a symmetric probabilistic allocation rule, such as the Shapley value, is partnership dissolution-proof on the class of convex games ${ }^{5}$ other well-known core allocation rules are neither partnership formation- nor dissolution-proof.

In section 5 we address the influence of the definition of stability on the results presented in section 3 and 4 .

In section 6 we explore the situation where several disjoint partnerships exist within a population and consider whether more players could be expected to join a partnership or if players have incentives to dissolve existing partnerships and possibly create new ones. A few results on the stability of partnership structures are provided. We comment on the consequence of applying different definitions of stability in this context. Section 7 concludes.

\section{Partnerships: model and definitions}

A cooperative game with side-payments is a pair $(N, v)$, where $N=\{1, . ., n\}$ is a finite set of players with $|N|=n \geq 3$ and $v$ is a mapping from $2^{N}$ into $\mathbb{R}_{+}$, with $v(\varnothing)=0{ }^{6}$ Note that we consider only non-negative coalition worths. Since $N$ is fixed we refer to a game $(N, v)$ simply as $v$, when no confusion can arise. Also, for players $i$ and $j$, we write $v(i)$ instead of $v(\{i\}), v(i, j)$ instead of $v(\{i, j\})$ etc. Coalitions of players are subsets $S, T, Q \ldots$ of $N$. Given a vector $x \in \mathbb{R}^{N}, x(S)$ specifies the aggregate payoff $\sum_{i \in S} x_{i}$ of coalition $S \subseteq N$. An allocation rule for a family of games $\mathcal{V}$ is a function $\phi: \mathcal{V} \rightarrow \mathbb{R}^{N}$ such that $\sum_{i \in N} \phi_{i}(v)=v(N)$, i.e., it satisfies efficiency.

The core of a game $v$ is the set $C(v)=\left\{x \in \mathbb{R}^{N} \mid x(N)=v(N), x(S) \geq v(S)\right.$ for all $S \subset N\}$. A game $v$ is balanced if $C(v) \neq \varnothing$, monotonic if $v(S) \leq v\left(S^{\prime}\right)$ for all coalitions $S, S^{\prime} \subseteq N$ with $S \subseteq S^{\prime}$, superadditive if $v(S)+v\left(S^{\prime}\right) \leq v\left(S \cup S^{\prime}\right)$ for any disjoint coalitions $S, S^{\prime} \subseteq N$, and convex if $v(S)+v\left(S^{\prime}\right) \leq v\left(S \cap S^{\prime}\right)+v\left(S \cup S^{\prime}\right)$ for all coalitions $S, S^{\prime} \subseteq N$. Convexity implies both superadditivity and balancedness, cf.

\footnotetext{
${ }^{5}$ Convex games hold the property that a player's marginal contribution to a coalition (weakly) increases as the coalition grows.

${ }^{6}$ For a general treatment of cooperative games, see, e.g., Owen (1995) or Peleg and Sudhölter (2003).
} 
Shapley (1971). Note also that for convex games non-negativity implies monotonicity.

Players $i, j \in N$ are said to be symmetric in $v$ if for all $S \subseteq N \backslash\{i, j\}$ it holds that $v(S \cup\{i\})=v(S \cup\{j\})$. An allocation rule $\phi$ is symmetric if symmetric players are treated equally, that is, if $\phi_{i}(v)=\phi_{j}(v)$ for all symmetric players $i, j \in N$ in $v$. We say that player $i$ is a null player in the game $v$ if $v(S \cup\{i\})=v(S)$ for all $S \subseteq N \backslash\{i\}$, and that an allocation rule $\phi$ satisfies the null player condition (or briefly, is null) if $\phi_{i}(v)=0$ whenever $i$ is a null player.

A coalition $T \subseteq N$ forms a partnership when each player in $T$ commits to not contributing to any coalition $S$ for which $T \nsubseteq S$. More precisely, we follow Kalai and Samet (1987), and Carreras (1996) and say that a coalition $T \subseteq N$ is a partnership in $v$ if

$$
v(R \cup S)=v(R) \text { for all } S \subset T \text { and all } R \subseteq N \backslash T \text {. }
$$

This definition of a partnership corresponds to the notion of a p-type coalition introduced in Kalai and Samet (1987) and to what Hart and Moore (1990) call a joint ownership, see also Carreras (1996) and Segal (2003, p. 447). ${ }^{7}$ As in Carreras (1996), the creation of a partnership changes the game from $(N, v)$ to $\left(N, v^{T}\right)$ defined by

$$
v^{T}(S)=\left\{\begin{array}{c}
v(S), \text { if } T \subseteq S \\
v(S \backslash T), \text { otherwise. }
\end{array}\right.
$$

Notice that in the partnership game, $v^{T}$, of $v$ any coalition $S \subset T$ has the same worth as the empty coalition, i.e. $v^{T}(S)=0$. Further, all players in $T$ are symmetric in $v^{T}$. As mentioned above, we restrict attention to the class of non-negative games. This allows us to preserve the natural interpretation of partnership formation and disregard situations where the formation of a partnership allows the worth of a coalition within the partnership to increase from some negative amount to zero.

\footnotetext{
${ }^{7}$ We follow the line of literature defining a partnership as a property of a coalition with respect to the game. In contrast, Reny et al. (2009) consider the "partnership property" which is a property of a collection of coalitions and does not involve the game but plays a role for their study of allocations of a game for which there are no asymmetric dependencies between any two players.
} 
Given an allocation rule $\phi$, it is not profitable to create any partnership if and only if

$$
\sum_{i \in T} \phi_{i}\left(v^{T}\right) \leq \sum_{i \in T} \phi_{i}(v)
$$

for all $T \subseteq N$ and all $v$. Allocation rules that satisfy this condition will be called partnership formation-proof. Note that the definition implies that redistribution of the joint profit is possible among members of a partnership. If the reverse inequality always holds we say that the allocation rule is partnership dissolution-proof. For convenience we use the abbreviations PFP and PDP respectively throughout this paper.

Lemma 1 below states that the partnership game inherits any properties such as non-negativity, monotonicity, superadditivity, balancedness, and convexity from the original game. The proofs are straightforward for non-negativity, monotonicity, and superadditivity (as pointed out in Carreras (1996)) and are omitted here. The proofs for the latter two properties are given.

Lemma 1 The following classes of games are closed under partnership formation.

\section{Non-negative}

2. Monotonic

\section{Superadditive}

\section{Balanced}

\section{Convex}

Proof: To show that a balanced game is closed under partnerships assume that $x \in$ $C(v)$. Note that since $v(i) \geq 0$ for all $i \in N$, we have $C(v) \subseteq \mathbb{R}_{+}^{N}$. For coalitions $S \subseteq N$ such that $T \subseteq S$ or $T \cap S=\varnothing$ we have $x(S) \geq v^{T}(S)$ since $v^{T}(S)=v(S)$. For coalitions $S \subseteq N$ such that $T \cap S \neq \varnothing$ and $T \nsubseteq S$, we also have $x(S) \geq v^{T}(S)$ since, if not, then $x(S \backslash T) \leq x(S)<v^{T}(S)=v(S \backslash T)$ by $C(v) \subseteq \mathbb{R}_{+}^{N}$, contradicting that $x \in C(v)$. Thus, $x \in C\left(v^{T}\right)$. 
Convex games are closed under partnerships if for any convex game $v$ and any $T$ it holds that $v^{T}$ is convex, i.e. for any two coalitions $S, S^{\prime} \subseteq N$ the inequality $v^{T}(S)+v^{T}\left(S^{\prime}\right) \leq v^{T}\left(S \cup S^{\prime}\right)+v^{T}\left(S \cap S^{\prime}\right)$ holds. If $T \subseteq S$ and $T \subseteq S^{\prime}$, the inequality is immediate from the convexity of $v$. If $T \nsubseteq \subseteq S$ and $T \nsubseteq S^{\prime}$, then

$$
\begin{aligned}
v^{T}(S)+v^{T}\left(S^{\prime}\right) & =v(S \backslash T)+v\left(S^{\prime} \backslash T\right) \\
& \leq v\left(\left(S \cup S^{\prime}\right) \backslash T\right)+v\left(\left(S \cap S^{\prime}\right) \backslash T\right) \\
& \leq v^{T}\left(S \cup S^{\prime}\right)+v^{T}\left(S \cap S^{\prime}\right),
\end{aligned}
$$

where the first inequality follows from convexity of $v$, and the second follows since $v\left(\left(S \cap S^{\prime}\right) \backslash T\right)=v^{T}\left(S \cap S^{\prime}\right)$ and $v^{T}\left(S \cup S^{\prime}\right)=v\left(\left(S \cup S^{\prime}\right) \backslash T\right)$ if $T \nsubseteq \subseteq \cup S^{\prime}$ and $v^{T}\left(S \cup S^{\prime}\right)=v\left(S \cup S^{\prime}\right) \geq v\left(\left(S \cup S^{\prime}\right) \backslash T\right)$ if $T \subseteq S \cup S^{\prime}$ (because by monotonicity and convexity $v$ is monotonic).

If $T \subseteq S$ and $T \nsubseteq S^{\prime}$, then

$$
\begin{aligned}
v^{T}(S)+v^{T}\left(S^{\prime}\right) & =v(S)+v\left(S^{\prime} \backslash T\right) \\
& \leq v\left(S \cup S^{\prime}\right)+v\left(\left(S \cap S^{\prime}\right) \backslash T\right) \\
& =v^{T}\left(S \cup S^{\prime}\right)+v^{T}\left(S \cap S^{\prime}\right),
\end{aligned}
$$

where the inequality follows from the convexity of $v$. Since the remaining case $T \nsubseteq S$ and $T \subseteq S^{\prime}$ is symmetric, we conclude that $v^{T}$ is convex.

\section{Partnership formation- and dissolution-proofness}

It is easy to construct an allocation rule that is both PFP and PDP. Consider as a trivial example an allocation rule that always allocates the total worth of the grand coalition to the same player, i.e., a dictatorial rule. Since the worth that is allocated to some coalition $S \subseteq N$ will be unchanged in any partnership game the dictatorial rule is both PFP and PDP. There also exists a symmetric allocation rule $\phi$ that is both PFP 
and PDP: the equal-split rule $\phi^{E S}$ defined by $\phi_{i}^{E S}(v)=\frac{v(N)}{n}$ for all $v$ and all $i \in N$. It is, in fact, the only symmetric rule that is both $\mathrm{PFP}$ and $\mathrm{PDP}{ }^{8}$

Proposition 1 For any class of games that is closed under partnerships, there is one and only one symmetric PFP and PDP allocation rule: the equal split rule.

Proof: It is clear that the equal split rule is PFP and PDP. We show that it is the only rule that satisfies both properties. Suppose that a symmetric rule $\phi$ is PFP and PDP and $\phi \neq \phi^{E S}$. Thus there is a game $v$ such that $\phi(v) \neq \phi^{E S}(v)$. Pick a player $i_{\text {min }} \in N$ for which no other player gets a smaller payoff at the allocation $\phi(v)$, and pick a player $i_{\max } \in N$ for which no other player gets a larger payoff at $\phi(v)$.

First, let $T=N \backslash\left\{i_{\min }\right\}$ and consider the partnership game $v^{T}$ based on $v$. Since $\phi$ is both PFP and PDP, we have $\sum_{i \in T} \phi_{i}\left(v^{T}\right)=\sum_{i \in T} \phi_{i}(v)$, and hence $\phi_{i_{\min }}\left(v^{T}\right)=\phi_{i_{\min }}(v)$. In particular we have by symmetry that $\phi_{i_{\max }}\left(v^{T}\right)=\frac{\sum_{i \in T} \phi_{i}\left(v^{T}\right)}{|T|}>\frac{v(N)}{|N|}$. Note that $v^{T}(S)=v(S)$ for $S=T, v^{T}(S)=v\left(i_{\min }\right)$ for any coalition $S \ni i_{\min }, S \neq N$, and $v^{T}(S)=0$ otherwise.

Second, let $U=N \backslash\left\{i_{\max }\right\}$ and consider the partnership game $v^{T U}$ based on $v^{T}$. (We write $v^{T U}$ rather than $\left(v^{T}\right)^{U}$ to avoid cumbersome notation). Since $\phi$ is both PFP and PDP, we have $\sum_{i \in U} \phi_{i}\left(v^{T U}\right)=\sum_{i \in U} \phi_{i}\left(v^{T}\right)$, and hence $\phi_{i_{\max }}\left(v^{T U}\right)=\phi_{i_{\max }}\left(v^{T}\right)>$ $\phi_{i_{\min }}\left(v^{T}\right)$. In particular by symmetry: $\phi_{i_{\min }}\left(v^{T U}\right)=\frac{\sum_{i \in U} \phi_{i}\left(v^{T U}\right)}{|U|}<\frac{v(N)}{|N|}$. Note that $v^{T U}(S)=v\left(i_{\min }\right)$ if $S=U, S \neq N$ and $v^{T U}(S)=0$ for all $S \neq U, N$.

Third, consider again the coalition $T=N \backslash\left\{i_{\min }\right\}$ and the partnership game $v^{T U T}$ based on $v^{T U}$. Since $v^{T U}\left(i_{\min }\right)=0$ and $v^{T U}(T)=0$ we have $v^{T U T}(S)=0$ for all $S \neq N$. In particular, the game is symmetric. But since $\phi$ is $\mathrm{PFP}$ and $\mathrm{PDP}$ we have $\sum_{i \in T} \phi\left(v^{T U T}\right)=\sum_{i \in T} \phi\left(v^{T U}\right)$, and hence $\phi_{i_{\min }}\left(v^{T U T}\right)=\phi_{i_{\min }}\left(v^{T U}\right)<\frac{v(N)}{|N|}$, contradicting that $\phi$ is symmetric.

The equal split rule violates the null player condition. Thus, as a consequence of Proposition 1 we get the following negative result:

\footnotetext{
${ }^{8}$ After the initial version of this paper was submitted for publication, it has come to our attention that a similar result has recently and independently been found by van den Brink (2009).
} 
Corollary 1 For no class of games that is closed under partnerships, does there exist a symmetric allocation rule that is both PFP and PDP and satisfies the null player condition.

It is now natural to ask whether symmetric allocation rules exist that are either PFP or PDP while satisfying the null player condition. We get the following:

Proposition 2 i) For the class of non-negative convex games, there exist no symmetric PFP allocation rule satisfying the null player condition. ii) There exist no symmetric PDP allocation rule satisfying the null player condition on the class of non-negative balanced games.

Proof: For the first part suppose that $\phi$ is a PFP allocation rule. Let $n \geq 3$ and consider the (convex) game $v$ with $v(N)=v(N \backslash\{1\})=1$ and $v(S)=0$ otherwise. The only allocation consistent with symmetry and the null player condition is $\phi_{1}(v)=$ $0, \phi_{i}(v)=\frac{1}{n-1}$ for all $i \in N \backslash\{1\}$. Now assume that players 1 and 2 form a partnership, $T=\{1,2\}$. This implies $v^{T}(N)=1$ and $v^{T}(S)=0$ otherwise. Then by symmetry $\phi_{i}\left(v^{T}\right)=\frac{1}{n}$ for all $i \in N$. Since $\phi_{1}(v)+\phi_{2}(v)=\frac{1}{n-1}<\frac{2}{n}=\phi_{1}\left(v^{T}\right)+\phi_{2}\left(v^{T}\right)$ this contradicts that $\phi$ is PFP.

For the second part, in order to provoke a counter example suppose that $\phi$ is PDP and consider the following (balanced) game $v$, where $n \geq 3 . v(N)=1, v(1)=1$, and $v(S)=0$ otherwise. If $T=\{1,2\}$ form a partnership the partnership game $v^{T}$ is symmetric implying that by symmetry $\phi_{i}\left(v^{T}\right)=\frac{1}{n}$ for all $i \in N$. For $\phi$ to be PDP it must therefore hold that $\phi_{1}(v)+\phi_{2}(v) \leq \frac{2}{n}$. Next consider instead the formation of a partnership $U=\{2, \ldots, n\}$. Then $v^{U}(N)=1, v^{U}(S)=v(S \backslash U)=v(1)=1$ for all $S$ where $1 \in S$, and $v(S)=0$ otherwise, implying that all players $2, \ldots, n$ are null. Thus $\phi_{2}\left(v^{U}\right)=\cdots=\phi_{n}\left(v^{U}\right)=0$ and for $\phi$ to be PDP it must therefore hold that $\phi_{2}(v)=\cdots=\phi_{n}(v) \leq 0$. Since we know that $\sum_{i=1}^{n} \phi_{i}(v)=1$ this implies that $\phi_{1}(v)=1-(n-1) \phi_{2}(v)$. Substituting this into the condition that $\phi_{1}(v)+\phi_{2}(v) \leq \frac{2}{n}$ in turn gives $\phi_{2}(v) \geq \frac{1}{n}$ which contradicts $\phi_{2}(v) \leq 0$. 
Note that for the first part where the counter example is a non-negative convex game the result holds by implication for the classes of monotonic, superadditive, and balanced games. Note also that the counter example used for the second part of Prop. 2 is not a monotonic game. This is no coincidence as monotonicity does allow for a symmetric PDP rule that satisfies the null player condition:

Proposition 3 There exist a symmetric PDP allocation rule satisfying the null player condition on the class of monotonic games. Indeed, the equal non-null split rule (which divides $v(N)$ equally between all non-null players in $N)$ satisfies PDP.

Proof: For a game $v$, let $D(v) \subseteq N$ denote the set of null players $i$ in $N$. Let $\phi^{*}$ be the rule that gives 0 to the null players and then divides $v(N)$ equally between the remaining players in $N$; i.e. $\phi_{i}^{*}(v)=0$ if $i \in D(v)$ and $\phi_{i}^{*}(v)=\frac{v(N)}{|N \backslash D(v)|}$ otherwise. Clearly, $\phi^{*}$ is a symmetric rule satisfying the null player condition. We now show that $\phi^{*}$ satisfies the PDP property.

For this, consider a game $v$ and suppose that the players in $T \subseteq N$ form a partnership. We now claim that (i) for all $i \notin T$ we have $i \in D(v)$ implies $i \in D\left(v^{T}\right)$, and (ii) for all $i \in T$ we have $i \notin D(v)$ implies $i \notin D\left(v^{T}\right)$.

Ad (i). Let $i \in D(v) \backslash T$. Let $S \subseteq N$ be an arbitrary coalition with $i \notin S$. If $S \cap T=\varnothing$ or $T \subseteq S$ we have $v^{T}(S)=v(S)$ and $v^{T}(S \cup\{i\})=v(S \cup\{i\})$, and thus $v^{T}(S \cup\{i\})-v^{T}(S)=v(S \cup\{i\})-v(S)=0$. If $T \nsubseteq S$ and $S \cap T \neq \varnothing$ then $v^{T}(S)=v(S \backslash T)$ and $v^{T}(S \cup\{i\})=v((S \backslash T) \cup\{i\})$, and thus have $v^{T}(S \cup\{i\})-v^{T}(S)=$ $v((S \backslash T) \cup\{i\})-v(S \backslash T)=0$. Thus, $i \in D\left(v^{T}\right)$.

Ad (ii). Let $i \in T \backslash D(v)$. Since $i$ is not a null player and the game $v$ is monotonic, there is a coalition $S \subseteq N$ with $i \notin S$ where $v(S \cup\{i\})-v(S)>0$. In particular, we have $v^{T}((S \cup T) \backslash\{i\})=v(S \backslash T) \leq v(S)$, and $v^{T}(S \cup T)=v(S \cup T) \geq v(S \cup\{i\})$ where the inequalities follow by monotonicity of $v$. We therefore get $v^{T}(S \cup T)-v^{T}((S \cup T) \backslash\{i\}) \geq$ $v(S \cup\{i\})-v(S)>0$. Thus, $i \notin D\left(v^{T}\right)$.

If $v(N)>0$ we have $N \backslash D(v) \neq \varnothing$ and since $v^{T}(N)=v(N)$ we get $N \backslash D\left(v^{T}\right) \neq \varnothing$. In particular, it follows by (i) and (ii) that $\frac{|T \backslash D(v)|}{|N \backslash D(v)|} \leq \frac{\left|T \backslash D\left(v^{T}\right)\right|}{\left|N \backslash D\left(v^{T}\right)\right|}$. Thus, $\sum_{i \in T} \phi_{i}^{*}\left(v^{T}\right)=$ 
$\frac{\left|T \backslash D\left(v^{T}\right)\right|}{\left|N \backslash D\left(v^{T}\right)\right|} v^{T}(N) \geq \frac{|T \backslash D(v)|}{|N \backslash D(v)|} v(N)=\sum_{i \in T} \phi_{i}^{*}(v)$ which shows that $\phi^{*}$ is PDP.

Proposition 2 ii) implies that there exists no symmetric PDP allocation rule satisfying the null player condition on the family of non-negative games. In fact, PDP allocation rules cannot be found on this family of games even if the symmetry requirement is dropped.

Proposition 4 There exist no PDP allocation rules satisfying the null player condition on the class of non-negative games.

Proof: In order to provoke a counter example, suppose that $\phi$ is PDP and consider the game $v$ defined by $n \geq 3, v(N)=1, v(i)=1$ for all $i, v(S)=0$ otherwise. There must exist some two player combination $\{i, j\}$ for which $\phi_{i}(v)+\phi_{j}(v)>0$. Then, if $T=\{i, j\}$ forms a partnership they become null players in $v^{T}$ with payoff $\phi_{i}\left(v^{T}\right)=\phi_{j}\left(v^{T}\right)=0$ contradicting that $\phi$ is $\mathrm{PDP}$.

\section{The Shapley value and other core allocation rules}

An allocation rule $\phi$ defined on the family of balanced games is a core allocation rule if $\phi(v) \in C(v)$ for all balanced $v$. Note that all core allocation rules satisfy the null player condition. It turns out that the positive result from Prop. 3 cannot be strengthened to the case of core allocation rules, at least if $n \geq 6$.

Proposition 5 For $n \geq 6$, there exists no symmetric PDP core allocation rule on the class of monotonic balanced games.

Proof: Suppose that $\phi$ is a PDP symmetric core allocation rule. Let $n=6$ and define a (monotonic balanced) game $v$ as follows: $v(i)=0$ for all $i, v(1,2)=v(1,3)=v(2,3)=$ $v(1,2,3)=v(4,5,6)=2, v(S)=2$ if $S$ contains at least two players in $\{1,2,3\}$ but not coalition $\{4,5,6\}$ or if $S$ contains coalition $\{4,5,6\}$ but no more than one of the players in $\{1,2,3\}, v(S)=4$ if $S$ contains $\{4,5,6\}$ and at least one of the coalitions $\{1,2\},\{1,3\},\{2,3\}, v(N)=5$ and otherwise $v(S)=0$. Then $C(v)$ only contains 
one symmetric element and we must have $\phi(v)=\left\{1,1,1, \frac{2}{3}, \frac{2}{3}, \frac{2}{3}\right\}$. Let $T=\{1,2,3\}$. Then $v^{T}(S)=0$ if $|S| \leq 2, v^{T}(S)=2$ if contains either $\{1,2,3\}$ or $\{4,5,6\}$ but not both of them. By symmetry, we have $\phi_{\{1,2,3\}}\left(v^{T}\right)=\phi_{\{4,5,6\}}\left(v^{T}\right)=\frac{5}{2}<\phi_{\{1,2,3\}}(v)=3$ contradicting that $\phi$ is $\mathrm{PDP}$.

Finally, we note that since $\phi$ is a core allocation rule it satisfies the null player condition. Hence we can extend the counter example to any $n>6$ by adding null players.

As stated in Prop. 5 it is not possible to find symmetric PDP core allocation rules on the family of monotonic balanced games. It is, however, possible to find symmetric PDP core allocation rules on the family of convex games. Segal (2003) gives a condition for a partnership to always be (weakly) (un)profitable when a game is solved by a probabilistic value. It can be inferred from his analysis that partnerships are always (weakly) profitable in convex games. We give a short and illustrative proof for the case of the Shapley value. The Shapley value, which is a symmetric probabilistic value, is defined as

$$
\phi_{i}^{S h}(N, v)=\sum_{S \subseteq N, S \ni i} p(S)(v(S)-v(S \backslash\{i\})),
$$

where $p(S)=\frac{(|S|-1) !(|N|-|S|) !}{|N| !}$, cf. Shapley (1953).

Proposition 6 The Shapley value (which is indeed a core allocation rule on convex games) is a symmetric PDP core allocation rule on the class of non-negative convex games.

Proof: For any $n \geq 3$ and $i \notin T$, we have

$$
\begin{aligned}
\phi_{i}^{S h}\left(v^{T}\right)= & \sum_{S \subseteq N, i \in S} p(S)\left[v^{T}(S)-v^{T}(S \backslash\{i\})\right] \\
= & \sum_{\substack{S \subseteq N, i \in S, T \subseteq S}} p(S)[v(S)-v(S \backslash\{i\})]+\sum_{\substack{S \subseteq N, i \in S, T \cap S=\varnothing}} p(S)[v(S)-v(S \backslash\{i\})]+ \\
& \sum_{\substack{S \subseteq N, i \in S, T \cap S \neq \varnothing, T \pm S}} p(S)[v(S \backslash T)-v(S \backslash(T \cup\{i\}))] .
\end{aligned}
$$


The value for player $i \notin T$ in the games $v$ and $v^{T}$ respectively will only differ in the last term. That is,

$$
\phi_{i}^{S h}\left(v^{T}\right)-\phi_{i}^{S h}(v)=\sum_{\substack{S \subseteq N, i \in S, T \cap S \neq \varnothing, T \nsubseteq S}} p(S)[v(S \backslash T)-v(S \backslash(T \cup\{i\}))-(v(S)-v(S \backslash\{i\}))]
$$

By convexity of $v$ we have $v(S \backslash T)-v(S \backslash(T \cup\{i\})) \leq v(S)-v(S \backslash\{i\})$ implying $\phi_{i}^{S h}\left(v^{T}\right)-\phi_{i}^{S h}(v) \leq 0$ for all $i \notin T$ and thus by efficiency $\sum_{i \in T} \phi_{i}^{S h}\left(v^{T}\right) \geq \sum_{i \in T} \phi_{i}^{S h}(v)$. Furthermore, in the case of strict convexity we have $\sum_{i \in T} \phi_{i}^{S h}\left(v^{T}\right)>\sum_{i \in T} \phi_{i}^{S h}(v)$.

As a corollary of Prop. 6 and Lemma 1 it can be noted that when the Shapley value is applied to strictly convex games some set of players can jointly profit from forming a partnership as long as the game is not symmetric in which case further partnership formation has no effect.

The Shapley value was shown to be a PDP core allocation rule on convex games, however, other well-known symmetric core allocation rules do not share this property as will be shown below.

Fujishige (1980) and Dutta and Ray (1989) and numerous subsequent papers have analyzed the allocation rule that for any convex game selects the unique most egalitarian allocation in the core. This rule will be denoted the Fujishige-Dutta-Ray allocation rule, $\phi^{F D R}$, in the following. The algorithm resulting in $\phi^{F D R}$ in a convex game partitions the set of players $N$ in a game $(N, v)$ into subsets $S_{1}, S_{2}, \ldots, S_{m}$, where $S_{1}$ is the (unique) largest coalition having the highest average worth in $(N, v)$. For any coalition $S$ and any characteristic function $v$ the average worth of $S$ under $v$ is defined by $e(S, v)=v(S) /|S|$. For $k=2, \ldots, m, S_{k}$ is the unique largest coalition with the highest average worth in the game $\left(N_{k}, v_{k}\right)$ with player set $N_{k}=N \backslash\left\{S_{1} \cup \cdots \cup S_{k-1}\right\}$, given that the worth of a coalition $S$ in any game $\left(N_{k}, v_{k}\right)$ is defined as $v_{k}(S)=v_{k-1}\left(S_{k-1} \cup S\right)-v_{k-1}\left(S_{k-1}\right)$, where $v_{1}=v$, see Dutta and Ray (1989). Then the amount allocated to a player $i$ according to $\phi^{F D R}$ equals $\phi_{i}^{F D R}=e\left(S_{k}, v_{k}\right)$ for all $i \in S_{k}$. In convex games $\phi^{F D R}$ is the 
unique egalitarian allocation and belongs to the core.

Another well-known allocation rule with favorable properties on the class of convex games is the nucleolus introduced by Schmeidler (1969). The nucleolus is the allocation rule $\phi^{n u}$ that assigns an allocation $x=\phi^{n u}(v)$ to each game $v$ such that $x$ lexicographically minimizes the vector of excesses $e(S, x)=v(S)-\sum_{i \in S} x_{i}$. The nucleolus is unique and is in the core whenever the core is non-empty. ${ }^{9}$

While it follows from Prop. 2 i) that none of the above-mentioned core allocation rules are PFP on convex games, it can be shown by way of simple counter examples that neither the Fujishige-Dutta-Ray allocation rule nor the nucleolus is PDP on the class of convex games.

Proposition 7 Neither the Fujishige-Dutta-Ray allocation rule nor the nucleolus is PDP on the class of non-negative convex games.

Proof: For $n=3$ consider a game $v$ defined by $v(N)=2, v(1)=1, v(1,2)=v(1,3)=$ 1 and $v(S)=0$ otherwise. Then $\phi^{F D R}(v)=\left(1, \frac{1}{2}, \frac{1}{2}\right)$ and $\phi^{n u}(v)=\left(\frac{4}{3}, \frac{1}{3}, \frac{1}{3}\right)$. If a partnership is formed between players 1 and 2 the game changes to $v^{T}(N)=2$, $v^{T}(1,2)=1$, and $v(S)=0$ otherwise. This implies $\phi^{F D R}\left(v^{T}\right)=\left(\frac{2}{3}, \frac{2}{3}, \frac{2}{3}\right)$, which shows that $\phi^{F D R}$ cannot be PDP since $\phi_{1}^{F D R}(v)+\phi_{2}^{F D R}(v)=\frac{3}{2}>\frac{4}{3}=\phi_{1}^{F D R}\left(v^{T}\right)+\phi_{2}^{F D R}\left(v^{T}\right)$. Likewise we get that $\phi^{n u}\left(v^{T}\right)=\left(\frac{3}{4}, \frac{3}{4}, \frac{1}{2}\right)$ from which we conclude that $\phi^{n u}$ cannot be $\operatorname{PDP}$ since $\phi^{n u}(v)+\phi^{n u}(v)=\frac{5}{3}>\frac{3}{2}=\phi^{n u}\left(v^{T}\right)+\phi^{n u}\left(v^{T}\right)$.

Since the FDR allocation rule as well as the nucleolus satisfy the null player condition similar counterexamples can be constructed for $n>3$ by adding null players to the game.

\footnotetext{
${ }^{9}$ It has been shown by Maschler, Peleg and Shapley (1971) that the nucleolus coincides with the prekernel on the domain of convex games.
} 


\section{Weak partnership formation- and dissolution- proofness}

The definition of PFP and PDP applied in sections 3 and 4 holds an implicit assumption of the ability of members in a partnership to redistribute the joint profit. An obvious alternative to this definition is to consider a deviation to be profitable only if it is individually profitable for every member of a partnership in question. Thus, we define an allocation rule $\phi$ to be weakly partnership formation-proof (WPFP) if and only if for any game $v$ and any nonempty $T \subseteq N$ there exists $i \in T$ such that $\phi_{i}^{T}(v) \leq \phi_{i}(v)$. Likewise, an allocation rule $\phi$ is said to be weakly partnership dissolution-proof (WPDP) if and only if for any game $v$ and any nonempty $T \subseteq N$ there exists $i \in T$ such that $\phi_{i}^{T}(v) \geq \phi_{i}(v)$. In the following we reconsider some of the previous results in light of these alternative definitions.

We consider again the equal non-null split rule $\phi^{*}$ and show that it is both WPFP and WPDP on the class of non-negative convex games. This finding contrasts Prop. 1.

Proposition 8 i) The equal non-null split rule $\phi^{*}$ is both WPDP and WPFP on the class of non-negative convex games, however, ii) There exists no symmetric WPFP rule satisfying the null player condition on the class of monotonic games.

Proof: For the first part: As in the proof of Proposition 3, let $D(v)$ denote the set of null players in $v$ and let $\phi_{i}^{*}(v)=0$ if $i \in D(v)$ and $\phi_{i}^{*}(v)=\frac{v(N)}{|N \backslash D(v)|}$ otherwise. Note that since $v$ is convex, $i \in N \backslash D(v)$ if and only if $v(N)-v(N \backslash\{i\})>0$. Consequently, for any partnership such that $T \subseteq N \backslash D(v)$, it follows that $\left|N \backslash D\left(v^{T}\right)\right|=|N \backslash D(v)|$ and the allocation remains unchanged, i.e. no one can profit from this type of deviation. However, for any partnership $T$ such that $T \nsubseteq N \backslash D(v)$ we get $\left|N \backslash D\left(v^{T}\right)\right|>|N \backslash D(v)|$ and $\phi_{i}^{*}\left(N, v^{T}\right)<\phi_{i}^{*}(N, v)$ for all $i \in N \backslash D(v)$, implying that forming a partnership can never be strictly profitable for each player in $T$. Thus, the allocation rule is WPFP. On the other hand, when $T \nsubseteq N \backslash D(v)$ and $\left|N \backslash D\left(v^{T}\right)\right|>|N \backslash D(v)|$ then for those $i \notin N \backslash D(v)$ but in $T$ (and thus in $N \backslash D\left(v^{T}\right)$ ) we have that $\phi_{i}^{*}(v)=0<\frac{v(N)}{\left|N \backslash D\left(v^{T}\right)\right|}=$ 
$\phi_{i}^{*}\left(N, v^{T}\right)$. Since these players will prefer to keep the partnership intact the allocation rule will also be WPDP.

For the second part, let $N=\{1, \ldots, n\}$ and consider the (monotonic) game $v$ where $v(S)=0$ if $|S|=1$ and $v(S)=1$ otherwise (such that $v(i, j)-v(i)=1$ for $j \neq i$ and $v(S)-v(S \backslash\{i\})=0$ otherwise). Let $\phi$ be a symmetric rule satisfying the null player condition. Then $\phi_{i}(v)=\frac{1}{n}$ for all $i \in N$. Now, let $T=\{1, \ldots, n-1\}$ form a partnership. Then, $v^{T}(T)=v^{T}(N)=1$ and and $v^{T}(S)=0$ otherwise. Since player $n$ is a null player in $v^{T}$ by symmetry we get $\phi_{i}\left(v^{T}\right)=\frac{1}{n-1}$ for all $i \in T$, contradicting WPFP.

From Prop. 3 we know that the equal non-null split rule (which is a a symmetric rule satisfying the null player condition) is also WPDP on the class of monotonic games. Further, the result from Prop. 5 holds also for the case of WPDP, that is, there exists no symmetric WPDP core allocation rule on the family of monotonic balanced games. To see this note that in the example given in the proof of Prop. 5, every player in the partnership is strictly better off by dissolving the partnership, implying that no WPDP allocation rule can be found.

Reconsidering the allocation rules from the previous section we notice first that since the Shapley value is PDP it is also WPDP, but as shown below it is not WPFP. However, the FDR-allocation rule is WPFP. Recall that on convex games the allocation rule $\phi^{F D R}$ satisfies the properties that $\phi_{i}^{F D R}=\phi_{j}^{F D R}$ for all $i, j \in S_{t}$ and $t=1, . ., m$ and that $\phi_{i}^{F D R}>\phi_{j}^{F D R}$ if $i \in S_{k}, \quad j \in S_{t}$ and $k<t$, cf. Dutta and Ray (1989).

Proposition 9 i) the FDR-allocation rule (which is indeed a symmetric allocation rule satisfying the null player condition) is WPFP on the class of non-negative convex games, however, ii) Neither the Shapley value nor the nucleolus is WPFP on non-negative convex games.

Proof: For the first part consider a game $(N, v)$ and let $N$ be partitioned into subsets $S_{1}, \ldots, S_{m}$ according to the description of the FDR-allocation rule in the previous section. Then $i \in S_{1}$ belongs to the coalition with the highest average worth and $\phi_{i}^{F D R}\left(v^{\mathcal{B}}\right)=$ $v^{\mathcal{B}}\left(S_{1}\right) /\left|S_{1}\right|$. Since creating a partnership will not strictly increase the payoff of a 
coalition but may decrease the payoff of certain coalitions, players in $S_{1}$ can never strictly profit from joining a partnership. Since no player belonging to $S_{1}$ will form a partnership with players outside $S_{1}$ and no partnership among players in $N \backslash S_{1}$ can affect $v^{\mathcal{B}}\left(S_{1}\right)$, each player in of $S_{1}$ is secured the payoff $v^{\mathcal{B}}\left(S_{1}\right) /\left|S_{1}\right|$.

The algorithm first allocates the worth to $S_{1}$ and then considers the set of remaining players $N \backslash S_{1}$. Thus within the player set $N \backslash S_{1}$ the players in $S_{2}$ will be allocated the greatest worth among the remaining coalitions. Given this, the players of $S_{2}$ could never (strictly) profit from joining a partnership among the remaining players. This reasoning can be applied to any $S_{k}, k=1, . ., m$, in the partitioning of $N$. Since this holds for any $v$ it can be concluded that the FDR-allocation rule is WPFP.

For the second part: consider the (convex) game $v$ defined by $n=3, v(N)=$ $3, v(1)=1, v(2)=v(3)=0, v(1,2)=v(1,3)=2$ and $v(2,3)=1$. Then $\phi^{S h}(v)=$ $\phi^{n u}(v)=\left(\frac{5}{3}, \frac{2}{3}, \frac{2}{3}\right)$. If a partnership is formed between players 2 and 3 the game changes to $v(N)=3, v(1)=1, v(2)=v(3)=0, v(1,2)=v(1,3)=v(2,3)=1$. This implies $\phi^{S h}\left(v^{T}\right)=\left(\frac{4}{3}, \frac{5}{6}, \frac{5}{6}\right)$, which shows that $\phi^{S h}$ cannot be WPFP since $\phi_{2}^{S h}(v)=\phi_{3}^{S h}(v)=$ $\frac{2}{3}<\frac{5}{6}=\phi_{1}^{S h}\left(v^{T}\right)=\phi_{2}^{S h}\left(v^{T}\right)$. Likewise we get that $\phi^{n u}\left(v^{T}\right)=\left(\frac{6}{4}, \frac{3}{4}, \frac{3}{4}\right)$ from which we conclude that $\phi^{n u}$ cannot be WPFP since $\phi^{n u}(v)=\phi^{n u}(v)=\frac{2}{3}<\frac{3}{4}=\phi^{n u}\left(v^{T}\right)=$ $\phi^{n u}\left(v^{T}\right)$. Again similar examples can be constructed for $n>3$ by adding null players to the game.

Since the FDR rule satisfies symmetry and the null player condition it shows that Prop. 2 i) does not hold for the case of WPFP.

\section{Stability of partnership structures}

Until now we have considered the existence of PFP and/or PDP allocation rules on specific classes of games as well as the properties of certain allocation rules. In this context we considered the decision of a group of players to form or dissolve a given partnership. However, given a population where several disjoint partnerships may exist a related problem would be to consider the incentives of any group of players (from the 
same or from different partnerships) to form a new partnership, possibly breaking up others in the process. In other words we could consider the incentives of any group of players to change the partnership structure.

Consider a game $v$. Define any partition $\mathcal{B}=\left\{T_{1}, T_{2}, \ldots, T_{m}\right\}$ of $N$ as a partnership structure with $m$ partnerships and note that now we consider an element of $\mathcal{B}$ with only one member a partnership. The game $v^{\mathcal{B}}$ is then defined by $\left.v^{\mathcal{B}}=\left(\ldots\left(v^{T_{1}}\right)^{T_{2}}\right) \ldots\right)^{T_{m}}$ and the worth of a coalition $S$ is $v^{\mathcal{B}}(S)=v\left(\bigcup_{T_{j} \subseteq S} T_{j}\right) .{ }^{10}$ Given a game $v$ and an allocation rule $\phi$ we say that a partnership structure $\mathcal{B}$ is stable, if no set of players can profitably leave their respective partnerships and form new (possibly trivial) partnerships. In this context a change from one partnership structure $(\mathcal{B})$ to another $\left(\mathcal{B}^{\prime}\right)$ is considered profitable for a set of players $S$ if the payoff allocated to each player in the set is strictly larger under the new partnership structure, that is, if $\phi_{i}\left(v^{\mathcal{B}^{\prime}}\right)>\phi_{i}\left(v^{\mathcal{B}}\right)$ for all $i \in S$. Alternatively, one could apply a stronger notion of stability by considering a change of partnership structure to be profitable for a set of players $S$ whenever the total worth allocated to $S$ is greater under $\mathcal{B}^{\prime}$ than $\mathcal{B}$. While the latter definition is in accordance with the analysis of sections 3 and 4 , in this section we nevertheless choose to consider its weaker counterpart discussed in the previous section. First, this is the definition applied in other papers where the stability of partnership structures - or alternatively, coalition structures - have been analyzed, see e.g., Hart and Kurz (1983, 1984) and Segal (2003). ${ }^{11}$ Second, while the previous analysis concerned only the group decision of whether or not to form a partnership in a given game, the approach taken in the present section also considers an individual's incentives to leave an existing partnership and possibly join another. Concerning such decisions the strong version of stability may not be a satisfactory concept as the following example demonstrates.

Consider the game $(N, v)$ defined by $N=\{1,2,3\}, v(N)=4, v(1,2)=3$ and

\footnotetext{
${ }^{10}$ This definition is applicable also to the case where different partnerships do not necessarily consist of disjoint sets of players. As noted in Carreras (1996), the formation order of the partnerships does not matter.

${ }^{11}$ What we in the present paper have denoted "a partnership" is in the terminology of Segal (2003) referred to as a special case of "exclusion" or "exclusive integration".
} 
$v(S)=0$ otherwise. Note that for the trivial partnership structure $\mathcal{B}=\{\{1\},\{2\},\{3\}\}$ we have that $\left(N, v^{\mathcal{B}}\right)=(N, v)$. In this game the FDR allocation becomes $\phi^{F D R}\left(v^{\mathcal{B}}\right)=$ $\left(\frac{3}{2}, \frac{3}{2}, 1\right)$. If a partnership $T=\{1,3\}$ is created the partnership structure changes to $\mathcal{B}^{\prime}=\{\{1,3\},\{2\}\}$ and the game $\left(N, v^{\mathcal{B}^{\prime}}\right)$ is defined by $v^{\mathcal{B}^{\prime}}(N)=4$ and $v^{\mathcal{B}^{\prime}}(S)=0$ otherwise, implying that $\phi^{F D R}\left(v^{\mathcal{B}^{\prime}}\right)=\left(\frac{4}{3}, \frac{4}{3}, \frac{4}{3}\right)$. Since the change from $\mathcal{B}$ to $\mathcal{B}^{\prime}$ is jointly profitable for the players in the partnership the trivial partnership structure cannot be (strongly) stable. However, since $\phi_{1}^{F D R}\left(v^{\mathcal{B}^{\prime}}\right)=\frac{4}{3}<\frac{3}{2}=\phi_{1}^{F D R}\left(v^{\mathcal{B}}\right)$ player 1 as an individual was strictly better off under $\mathcal{B}$ and will therefore have incentives to leave the partnership he just joined. The problem is that when considering the strong version of stability we allow a player to form a partnership even if he is individually worse off assuming that other members of the partnership will be able to compensate him for joining. However, when considering his decision to leave the partnership we only evaluate the payoff allocated to this player individually according to the allocation rule and thus fail to take the possible compensation offered by other partnership members into account (i.e., the redistribution of the joint profit). To avoid this problem we hereafter only consider the weaker notion of stability.

The definition of stability of partnership structures may also depend on what happens when one player (or more) leaves a partnership that is, whether the entire partnership breaks down or a partnership continues to exist among the remaining partners. In Hart and Kurz (1983) this question leads to the definition of two distinct types of stability. For the extent of this paper we assume that in the case of some member(s) leaving a partnership the remaining members will continue to cooperate. The following results, however, apply to both settings.

Considering the stability of partnership structures a relevant question is whether a stable partnership structure always exists when employing a specific allocation rule. We find that for the case of the Fujishige-Dutta-Ray allocation rule a stable partnership structure does always exist. In fact when using the FDR allocation rule the trivial partnership structure is always stable for convex games. 
Corollary 2 For the class of non-negative convex games the trivial partnership structure consisting of singletons is stable for any game $(N, v)$ when the Fujishige-Dutta-Ray allocation rule is applied.

Proof: This follows directly from Prop. 8 i).

For both the Shapley value and the nucleolus examples of games where stable partnership structures exist can be found. Consider e.g. the example from Prop. 9 ii). Here the partnership structure $T^{\mathcal{B}}=\{\{1\},\{2,3\}\}$ is stable whether the allocation is done by the Shapley value or the nucleolus. ${ }^{12}$

However, for the Shapley value as well as the nucleolus there exist games for any $n \geq 3$ such that no stable partnership structure exist as shown below.

Proposition 10 For any $n \geq 3$, there exist non-negative convex games such that no stable partnership structure exists when the Shapley value $\phi^{\text {Sh }}$ is applied.

Proof: For any given $n \geq 3$, let the convex game $v$ be defined as follows: $v(N)=2$, $v(N \backslash\{i\})=1$ for all $i \neq 1$ and $v(S)=0$ otherwise. Further, for any partnership structure $\mathcal{B}=\left\{T_{1}, T_{2}, \ldots, T_{m}\right\}$ let $T^{\mathcal{B}}$ denote the set that is the union of player 1 and all players in some $T_{i}$ that is not a singleton. Then it holds for all $i \in T^{\mathcal{B}}$ that $v^{\mathcal{B}}(N \backslash\{i\})=0$.

Recalling the definition of the Shapley value and noting the structure of the game $v$, it is seen that the allocation to player $i$ will equal

$$
\phi_{i}^{S h}(v)=\frac{1}{n}\left(v(N)-v(N \backslash\{i\})+\sum_{S \ni i,|S|=n-1} \frac{1}{n(n-1)}(v(S)-v(S \backslash\{i\})) .\right.
$$

This implies that the worth allocated to player $i$ according to the Shapley value and a partnership structure $\mathcal{B}$ will be: $\phi_{i}^{S h}\left(v^{\mathcal{B}}\right)=\frac{1}{n} * 2+\frac{1}{n(n-1)}\left(n-\left|T^{\mathcal{B}}\right|\right)$ for $i \in T^{\mathcal{B}}$ and $\phi_{i}^{S h}\left(v^{\mathcal{B}}\right)=\frac{1}{n}+\frac{1}{n(n-1)}\left(n-\left|T^{\mathcal{B}}\right|-1\right)$ for $i \notin T^{\mathcal{B}}$, where the first term in each expression reflects the contribution made to the grand coalition and the second term reflects the contribution made to coalitions of size $n-1$.

\footnotetext{
${ }^{12}$ To see this note that for $T^{\mathcal{B}}=\{\{1,2\}, 3\}$ we get $\phi_{i}^{S h}(v)=\left(\frac{4}{3}, \frac{4}{3}, \frac{1}{3}\right)$ and $\phi_{i}^{S h}(v)=\left(\frac{5}{4}, \frac{5}{4}, \frac{1}{2}\right)$
} 
Now consider any $i \notin T^{\mathcal{B}}$ and let $\mathcal{B}^{\prime}$ denote some partnership structure such that $i \in$ $T^{\mathcal{B}^{\prime}}$ (i.e., where $i$ belongs to a non-trivial partnership). Then a change in the partnership structure from $\mathcal{B}$ to $\mathcal{B}^{\prime}$ would induce the following change in the Shapley value of player $i: \Delta \phi_{i}^{S h}\left(v^{\mathcal{B}^{\prime}}, v^{\mathcal{B}}\right)=\phi_{i}^{S h}\left(v^{\mathcal{B}^{\prime}}\right)-\phi_{i}^{S h}\left(v^{\mathcal{B}}\right)=\frac{1}{n}-\left(\left(\left|T^{\mathcal{B}^{\prime}}\right|-\left|T^{\mathcal{B}}\right|\right)-1\right) \frac{1}{n(n-1)}>0$, where $\left(\left|T^{\mathcal{B}^{\prime}}\right|-\left|T^{\mathcal{B}}\right|\right)$ reflects the change in the number of players belonging to a partnership. The change $\Delta \phi_{i}^{S h}\left(v^{\mathcal{B}^{\prime}}, v^{\mathcal{B}}\right)$ will always be positive (since $\left|T^{\mathcal{B}^{\prime}}\right|-\left|T^{\mathcal{B}}\right| \leq n-1$ ), and a player currently not in a partnership will therefore always have incentives to join one. Thus, if at least two players (other than player 1) are not in a (non-trivial) partnership they have incentives to form one.

On the other hand any player $i \in T^{\mathcal{B}}, T^{\mathcal{B}^{\prime}}$ is negatively affected when more players join partnerships. To see this note that in this case $\Delta \phi_{i}^{S h}\left(v^{\mathcal{B}^{\prime}}, v^{\mathcal{B}}\right)=-\left(\left|T^{\mathcal{B}^{\prime}}\right|-\right.$ $\left.\left.\left|T^{\mathcal{B}}\right|\right)\right) \frac{1}{n(n-1)}$ is negative whenever $\left|T^{\mathcal{B}^{\prime}}\right|>\left|T^{\mathcal{B}}\right|$. A player in $T^{\mathcal{B}}$ will therefore always prefer that fewer players belong to non-trivial partnerships. This can be interpreted as an incentive to exclude other players from partnerships or break up existing partnerships and form new and smaller ones. Therefore, if partnerships with more than two players exists, there will always be incentives to exclude one player. ${ }^{13}$ If partnerships with two players (other than player 1) exist, each member has incentives to break up the partnership and join a partnership with player 1, thereby excluding their former partner. However, if player 1 is in a two-player partnership he will have incentives to dissolve it.

Since the result shows non-existence of stability in the weak sense it also applies to the case where the stronger version of stability is invoked.

Proposition 11 For any $n \geq 3$, there exist non-negative convex games such that no stable partnership structure exists when the nucleolus $\phi^{\text {nu }}$ is applied.

Proof: For any given $n \geq 3$, let the convex game $v$ be defined as follows: $v(N)=2$, $v(N \backslash\{i\})=\frac{n}{n+2} v(N)=\frac{2 n}{n+2}$ for all $i \neq 1$ and $v(S)=0$ otherwise. As in the proof

\footnotetext{
${ }^{13}$ If remaining members were assumed to split up when some players leave a partnership (opposed to what is assumed here) there would be incentives to exclude more than one player.
} 
of Proposition 10 for any partnership structure $\mathcal{B}=\left\{T_{1}, T_{2}, \ldots, T_{m}\right\}$ let $T^{\mathcal{B}}$ denote the union of player 1 and the set of all players in some $T_{i}$ that is not a singleton. Then for any $n$ and any $\mathcal{B}$ the allocation according to the nucleolus will be:

$$
\begin{aligned}
& \phi^{n u}\left(v^{\mathcal{B}}\right)=\frac{2-\frac{2 n}{n+2}}{\left|T^{\mathcal{B}}\right|}+\frac{2}{n+2} \quad \text { for all } \quad i \in T^{\mathcal{B}}, \quad \text { and } \\
& \phi^{n u}\left(v^{\mathcal{B}}\right)=\frac{2}{n+2} \quad \text { for all } i \notin T^{\mathcal{B}} \text {. }
\end{aligned}
$$

First, to show this is true let

$$
e_{i j}=\max _{\substack{S \\ i \in S \\ j \notin S}}\left(v(S)-\sum_{i \in S} x_{i}\right)
$$

be the maximum excess over coalitions that contain $i$ but not $j$ where $x$ is the vector of allocations. Then since the nucleolus coincides with the prekernel on the domain of convex games it suffices to show that $e_{i j}=e_{j i}$ for all $i, j$ with $i \neq j$, cf. Maschler, Peleg and Shapley (1971).

For any $i \notin T^{\mathcal{B}}$ it holds that $v(i)-x_{i}=-\frac{2}{n+2}$ while $v(i)-x_{i}<-\frac{2}{n+2}$ for all $i \in T^{\mathcal{B}}$. Further, for all larger coalitions $S$ where $v(S)=0$ the excess must be even smaller. Thus the only coalitions left to consider are those coalitions where $v(S)>0$. For all coalitions $S=N \backslash\{i\}$ where $i \notin T^{\mathcal{B}}$ we get:

$$
\begin{aligned}
v(S)-\sum_{i \in S} x_{i} & =\frac{2 n}{n+2}-\left(2-\frac{2 n}{n+2}+\left|T^{\mathcal{B}}\right| \frac{2}{n+2}+\left(n-\left|T^{\mathcal{B}}\right|-1\right) \frac{2}{n+2}\right) \\
& =-\frac{2}{n+2} .
\end{aligned}
$$

Since all players in $T^{\mathcal{B}}$ are symmetric and all players not in $T^{\mathcal{B}}$ are symmetric we conclude that $e_{i j}=e_{j i}$ for all $i, j, i \neq j$. Specifically for any $i \notin T^{\mathcal{B}}, j \in N, j \neq i$ we have $e_{i j}=-\frac{2}{n+2}$ and for $i \in T^{\mathcal{B}}, j \notin T^{\mathcal{B}}$ we have $e_{i j}=-\frac{2}{n+2}$ while $e_{i j}=-\phi^{n u}\left(v^{\mathcal{B}}\right)$ for all $i \in T^{\mathcal{B}}, j \in T^{\mathcal{B}}, j \neq i$. The above allocation therefore equals the nucleolus.

Now, since the worth allocated to a player in $T^{\mathcal{B}}$ always exceeds the worth allocated 
to players not in $T^{\mathcal{B}}$ (no matter the number of players in $T^{\mathcal{B}}$ ) any two players not in a partnership will always have incentives to form one. On the other hand, considering two different partnership structures $T^{\mathcal{B}}, T^{\mathcal{B}^{\prime}}$ where $\left|T^{\mathcal{B}^{\prime}}\right|>\left|T^{\mathcal{B}}\right|$ it is seen that for a player $i \in T^{\mathcal{B}}, T^{\mathcal{B}^{\prime}}$ we have $\phi^{n u}\left(v^{\mathcal{B}^{\prime}}\right)<\phi^{n u}\left(v^{\mathcal{B}}\right)$. Therefore, if partnerships with more than two players exists, there will always be incentives for the members to exclude one player. ${ }^{14}$ If partnerships with two players (other than player 1) exist, each member has incentives to break up the partnership and join a partnership with player 1 to exclude their former partner. However, if player 1 is in a two-player partnership he will have incentives to dissolve it.

\section{Concluding remarks}

For several classes of games we have considered the existence of partnership formationproof and partnership dissolution-proof allocation rules. Such allocation rules will be immune to manipulation by players forming or dissolving partnerships. We showed that if allocations rules must satisfy symmetry and the null player condition then for some classes of games neither partnership formation-proof nor partnership dissolutionproof allocation rules exist, while dissolution-proof allocation rules that satisfy these properties do exist for other classes of games.

We considered in particular three well-known allocation rules; the Shapley value, the nucleolus and the Fujishige-Dutta-Ray rule. The first two are classical solution concepts that are widely used and studied in the literature while especially in recent years the egalitarian FDR-rule has attracted considerable attention.

We have focused here on non-negative games that are either convex or satisfy milder regularity conditions such a monotonicity, superadditivity and balancedness. Important cooperative decision problems, such a classes of common pool games, oligopoly games, production games and cost sharing situations, are indeed both non-negative

\footnotetext{
${ }^{14}$ If remaining members were assumed to split up when some players leave a partnership (opposed to what is assumed here) there would be incentives to exclude more than one player.
} 
and convex ${ }^{15}$. Thus, many cooperative game situations fall within the classes of games considered here.

When considering the incentives of players to form partnerships, a key distinction is whether partnerships are likely to form when every individual member is better off or simply when members are jointly better off. This leads to our distinction between partnership formation/dissolution-proofness and the weak versions of the concepts. The appropriate concept depends on assumptions of redistribution possibilities between members. These may vary greatly between different game situations and with the nature of the payoff (e.g. money, publicity, seats in a parliament, individual utility). For instance, if partnerships involve private firms establishing joint ownerships, or a couple getting married, redistribution between the partners is likely to take place unhindered. In contrast, in games when players are for example different regions in a country or different departments of a public institution, allocations are likely to be determined by a fixed rule and the possibilities for internal redistributions between players severely limited.

\section{References}

Aumann, R., Disadvantageous monopolies, Journal of Economic Theory, 6 (1973), 1-11.

Carreras, F., On the Existence and Formation of Partnerships in a Game, Games and Economic Behavior, 2 (1996), 54-67.

Carreras, F., Llongueras, M. and A. Magaña, On the Convenience to Form Coalitions or Partnerships in Simple Games, Annals of Operations Research, 137 (2005), 67-89.

Carreras, F., Llongueras, M. and M. Puente, Partnership formation and binomial semivalues, European Journal of Operational Research, 192 (2009), 487-499.

\footnotetext{
${ }^{15}$ See, for example, Shapley and Shubik (1969), Champsaur (1975), Curiel et al. 1989, Granot and Hojati (1990), Meinhardt (1999), Zhao (1999), Driessen and Meinhardt (2005).
} 
Champsaur, P., How to share the cost of a public good, International Journal of Game Theory, 4 (1975),113-129.

Charnes, A. and S. Littlechild, On the formation of unions in $n$-person games, Journal of Economic Theory, 10 (1975), 386-402.

Curiel, I., Pederzoli, G. and S. Tijs, Sequencing games, European Journal of Operational Research, 40 (1989), 344-351.

Derks, J. and S. Tijs, On Merge Properties of the Shapley Value, International Game Theory Review, 2 (2000), 249-57.

Driessen, THS. and H. Meinhardt H, Convexity of oligopoly games without transferable technologies, Mathematical Social Sciences, 50 (2005), 102-126.

Dutta, B. and D. Ray, A concept of egalitarianism under participation constraints, Econometrica, 57 (1989), 615-635.

Fujishige, S., Lexicographically optimal base of a polymatroid with respect to a weight vector, Mathematics of Operations Research,5 (1980), 186-196.

Granot, D. and M. Hojati, On cost allocation in communication networks, Networks, 20 (1990), 209-229.

Haller, H., Collusion Properties of Values, International Journal of Game Theory, 23 (1994), 261-281.

Hart, O. and J. Moore, Property rights and the nature of the firm, Journal of Political Economy, 98 (1990), 1119-1158.

Hart, S. and M. Kurz, Endogenous formation of coalitions, Econometrica, 51 (1983), 1047-1064.

Hart, S. and M. Kurz, Stable Coalition Structures, in: M. Holler, ed., Coalitions and Collective Action, Physica-Verlag, Wuerzburg, (1984), 235-258. 
Haviv, M., Consecutive amalgamations and an axiomatization of the Shapley value, Economics Letters, 49 (1995), 7-11.

Kalai, E. and D. Samet, On Weighted Shapley Values, International Journal of Game Theory, 16 (1987), 205-222.

Knudsen, P. H. and L.P. Østerdal, Merging and Splitting in Cooperative games: Some (Im)possibility Results, (2008). Available at www.econ.ku.dk/lpo.

Legros, P., Disadvantageous syndicates and stable cartels: the case of the nucleolus, Journal of Economic Theory, 42 (1987), 30-49.

Lehrer, E., An Axiomatization of the Banzhaf value, International Journal of Game Theory, 17 (1988), 89-99.

Maschler, M., Peleg, B. and L. Shapley, The kernel and bargaining set for convex games, International Journal of Game Theory, 1 (1971), 73-93.

Meinhardt, H., Common pool games are convex, Journal of Public Economic Theory, 1 (1999), 247-270.

Owen, G., Game Theory, Academic Press Orlando, Third Edition (1995).

Peleg, B. and P. Sudhölter, Introduction to the theory of cooperative games, Kluwer Academic Publishers, Boston, (2003).

Postlewaite, A. and R. Rosenthal, Disadvantageous syndicates, Journal of Economic Theory, 9 (1974), 324-326.

Reny, P.J., Winter E. and M. Wooders.The partnered core of a game with side payments. Working paper no. 09-W17, Department of Economics, Vanderbilt University. (2009)

Schmeidler, D., The nucleolus of a characteristic function game, SIAM J of Applied Mathematics, 17 (1969), 1163-1170. 
Segal, I., Collusion, Exclusion, and Inclusion in Random-Order Bargaining, Review of Economic Studies, 70 (2003), 439-460.

Shapley, L.S., A Value for n-person games, in: H. Kuhn and A.W. Tucker, eds., Contributions to the Theory of Games, Vol. II. Princeton University Press, (1953).

Shapley, L.S., Cores of convex games, International Journal of Game Theory, 1 (1971), $11-26$.

Shapley, L. and M. Shubik, On the core of an economic system with externalities, American Economic Review, 59 (1969), 678-684.

van den Brink, R., Efficiency and Collusion Neutrality of Solutions for Cooperative TU-Games, Tinbergen Institute Discussion Paper 09-065/1, (July 21, 2009).

Zhao, J., A necessary and sufficient condition for the convexity in oligopoly games, Mathematical Social Sciences, 37 (1999), 189-204. 